\title{
Triple Resonance Method for Molecular hfs Spectroscopy: Measurements on ${ }^{133} \mathrm{Cs}^{19} \mathrm{~F}^{*}$
}

\author{
Jens C. Zorn, David A. Stephenson, $\dagger$ J. Thomas Dickinson, and Thomas C. English. \\ Randall Laboratory of Physics, The Universily of Michigan, Ann Arbor, Michigan
}

(Received 29 June 1967)

\begin{abstract}
Three sequential oscillatory fields are employed in a molecular beam electric resonance spectrometer in order to observe molecular hfs transitions which do not obey the criteria for observability in the usual beam apparatus. The triple resonance method has been used to examine the hfs of CsF under conditions of very weak external fields. The method shows considerable promise for the detailed study of molecular hfs when more than one nucleus has a quadrupole moment, and for the observation of transitions at audio frequencies between closely spaced molecular energy levels.
\end{abstract}

The transitions which are observable in the conventional molecular beam electric resonance (MBER) spectrometer occur between those energy states which have different values of effective electric dipole moment in high field. We report here an experiment in which three, sequential oscillatory fields are employed to detect and measure transitions which do not obey this observability criterion. The additional spectral lines obtained in this way supplement the lines measured with a single oscillatory field and facilitate the understanding of the molecule's structure; moreover it is possible to measure directly many energy level separations which had previously been determined only as the difference between the frequencies of two spectral lines. The present experiment shows the use of a triple resonance method to measure hyperfine structure transitions in the $J=1, v=0$ state of ${ }^{133} \mathrm{Cs}^{19} \mathrm{~F}$ under conditions of very weak external fields, but the method is applicable to many molecules under a variety of field conditions.

\section{PRINCIPLES OF THE TRIPLE RESONANCE METHOD}

The zero-field energy level diagram of $\mathrm{CsF}$ in the $J=1$ state is shown in Fig. 1. The electric quadrupole coupling of the cesium nucleus (spin $I_{1}$ ) to the rotational angular momentum of the molecule $J$ is the dominant feature of the hyperfine structure; $I_{1}$ and $J$ couple to form $F_{1}$. The magnetic interaction of the fluorine nucleus splits each $F_{1}$ level into two $F$ levels, so the representation appropriate for very weak external field is $\left(J, I_{1}, F_{1}, I_{2}, F, M_{F}\right)$, abbreviated $\left(F_{1}, F\right)$. The effective electric dipole moment of the molecule in strong external field [where $\left(I_{1}, M_{1}, I_{2}, M_{2}, J, M_{J}\right)$ is a more suitable representation] is governed by $J,\left|M_{J}\right|$, so the trajectory of the molecule in the strong, inhomogeneous electric fields of the state selectors is dependent on $J,\left|M_{J}\right|$.

\footnotetext{
* Research supported in part by the U.S. Atomic Energy Commission.

$\dagger$ National Science Foundation predoctoral fellow.

$¥$ Present address: Department of Physics, Harvard University, Cambridge, Massachusetts.
}

In the $J=1$ state of $\mathrm{CsF}$ one finds ${ }^{1,2}$ that the $\left(F_{1}, F\right)$ levels $(7 / 2,3)$ and $(7 / 2,4)$ in very weak field correspond adiabatically to states in which $M_{J}=0$ in strong field, while the rest of the $J=1$ sublevels correspond to $\left|M_{J}\right|=1$; the transitions shown as $\alpha_{i}$ and $\beta_{i}(i=1, \cdots 4)$ on Fig. 1 are thus the only ones which are observable in very weak fields with the usual, single resonance MBER spectrometer. ${ }^{3}$ The selection rules ${ }^{4}$ which hold in very weak fields allow $\left|\Delta F_{1}\right|=0,1,2,|\Delta F|=0,1,2$, and $\left|\Delta M_{F}\right|=0,1$, however, and the triple resonance method described here permits the observation of all those transitions $\gamma_{i}$ which are allowed by the selection rules but which are not associated with a change of $\left|M_{J}\right|$ in high field.

These additional transitions proved quite useful in understanding the weak field hfs in CsF. As discussed in Ref. 1, the lines from excited vibrational states overlap the $v=0$ spectrum, making it difficult to identify the lines observed with the single resonance data. By performing triple resonance on this system the line assignments were strongly substantiated.

The beam spectrometer ${ }^{2}$ is one in which a $J=1$ molecule must be in the $M_{J}=0$ substate in both the $A$ and $B$ fields in order to be focused at the detector; molecules which are in the $\left(J, M_{J}\right)=(1, \pm 1)$ states in either the $A$ or $B$ fields (or both) are not detected. In what follows, the two focused substates which participate in the triple resonance are denoted by $a$ and $b$, and the two unfocused $J=1$ substates by $f$ and $g$. For $\mathrm{CsF}$ the $(7 / 2,3)$ and $(7 / 2,4)$ states are $a$ and $b$, as in Fig. 2, and if we want to measure the $(9 / 2,5)-(5 / 2,3)$ energylevel separation, for example, the latter two states will be $f$ and $g$.

Molecules leave the source and $A$ field to pass through rf regions 1,2 , and 3 before entering the $B$ field of the spectrometer. To simplify our illustration we assume (1) that the $A$ field state selector provides a

\footnotetext{
1 T. C. English and J. C. Zorn, J. Chem. Phys. 47, 3896 (1967), preceding paper.

${ }^{2}$ T. C. English, Ph.D. dissertation, University of Michigan (University Microfilms, Inc. Ann Arbor, 1966).

${ }^{3}$ P. Kusch and V. W. Hughes, Handbuch der Physik, S. Flügge, Ed. (Springer-Verlag, Berlin, 1959), Vol. 37/1, pp. 46-50, 138152 .
}

4 V. Hughes and L. Grabner, Phys. Rev. 79, 829 (1950). 
beam in which there are an equal number of molecules in states $a$ and $b$ but no molecules in states $f$ and $g$, and (2) that the action of each of the rf fields, when tuned to resonance, is to equalize the populations of the two states connected by the rf perturbation. These assumptions are for convenience in discussion and are not essential to the method.

The rf fields 1 and 3 are excited with fixed frequencies $\omega_{1}=\omega_{a f}$ and $\omega_{3}=\omega_{b \sigma}$ which are known from previous single-resonance measurements. Field 2 is excited with frequency $\omega_{2}$ which is varied over a range which includes $\omega_{f o}$. The sequence of events by which the $f-g$ transition may be detected is shown in Fig. 2 and proceeds as follows: In field 1 , state $f$ is populated at the expense of state $a$. If the frequency of the rf-exciting field 2 is equal to $\omega_{f g}$, there will be an appreciable number of molecules in state $g$ before the beam enters field 3 . In field 3 , where the $g-b$ transition occurs, the net transfer of

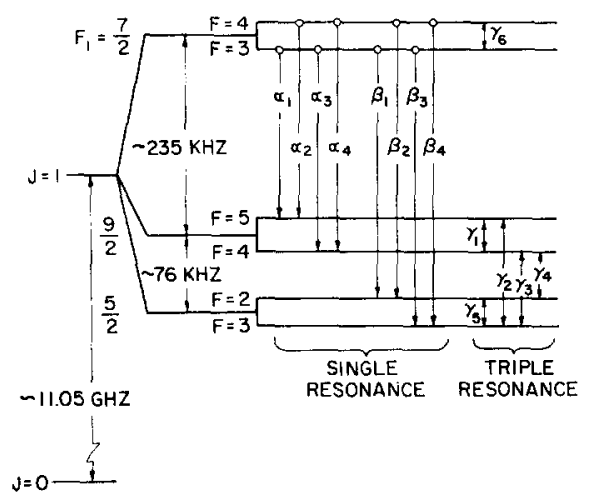

FIG. 1. Energy levels of the $J=1$ rotational state of ${ }^{133} \mathrm{Cs}^{19} \mathrm{~F}$ with no external field applied. The transitions $\alpha_{i}$ and $\beta_{i}$ can be seen by using a single if region; the transitions labeled $\gamma_{i}$ are seen only with the triple-resonance method.

molecules out of state $b$ depends on the population of $g$. It is the molecules which leave field 3 in states $a$ and $b$ which contribute to the detected signal, so the $\omega_{2}$ resonance can be detected by its influence on the population of state $b$.

As can be seen from analysis similar to that just presented, the occurrence of a resonance in rf field 2 can be detected with many other combinations of frequencies, as long as both $\omega_{1}$ and $\omega_{3}$ induce transitions which correspond to a change in $\left|M_{J}\right|$. For example, the $a-b$ transition can be observed if $\omega_{1}=\omega_{a f}, \omega_{2}=\omega_{a b}$, and $\omega_{3}=\omega_{a g}$. It can also be observed if $\omega_{1}=\omega_{a f}, \omega_{2}=\omega_{a b}$, and $\omega_{3}=\omega_{b f}$. In this latter case, the level $g$ is not needed so that the method can be used with three levels as well as with four.

There are similarities between the present experiment and the method developed by Woodgate and Sandars ${ }^{5}$ for the measurement of nuclear moments with an atomic beam magnetic resonance spectrometer.

G G. Woodgate and P. Sandars, Nature 181, 1395 (1958).

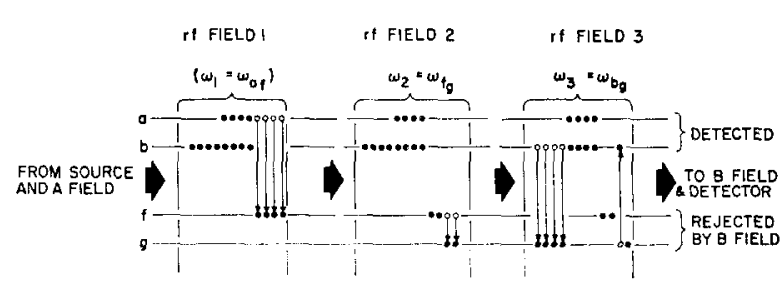

FIG. 2. Idealized operation of the triple-resonance method.

\section{EXPERIMENT}

The measurements are done with an MBER spectrometer, fully described elsewhere, ${ }^{2}$ in which the $C$ field is provided with three distinct regions where of can be applied. The construction of the $C$ field is such that the applied dc electric field is the same in all three $\mathrm{rf}$ regions. These rf regions are similar to (but shorter than) the rf field used for the single resonance measurements on $\mathrm{CsF}^{1}$ and $\mathrm{RbF}^{6}$ done with the same spectrometer. The first and third rf regions are each $10 \mathrm{~cm}$ long; the second rf field, in which the transitions of most interest are induced, is $30 \mathrm{~cm}$ long. The spacing between rf fields is $2 \mathrm{~cm}$. The operation of each $\mathrm{rf}$ region is individually tested by using it alone to measure a spectral line in the usual, single resonance fashion: linewidths very close to the expected values $(5 \mathrm{kHz}$ for the shorter fields, and $1.6 \mathrm{kHz}$ for rf field 2) are obtained, so there is assurance that the transition regions are operating in the expected way.

A tungsten-wire surface ionizer is used to detect the beam; the current from the ionized molecules is measured directly with a vibrating-reed electrometer. The four-pole, electrostatic $A$ and $B$ fields provide a beam

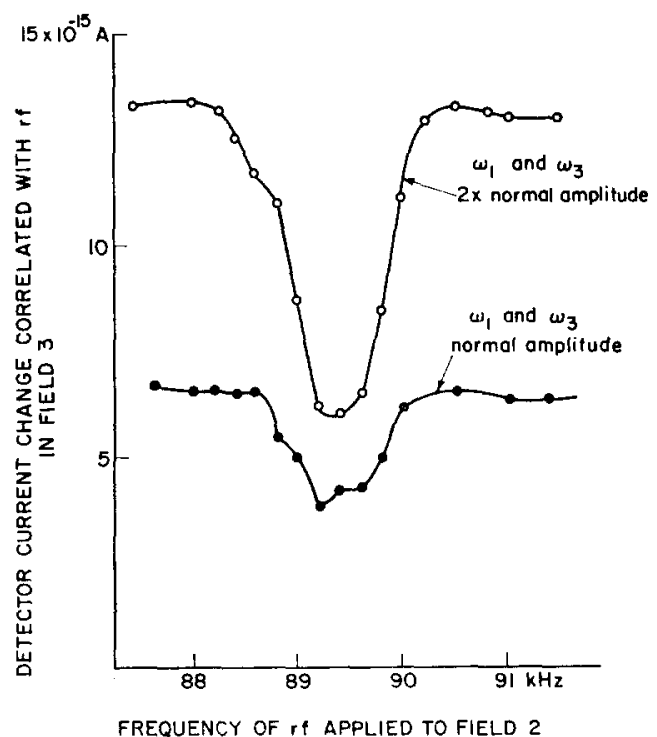

FIG. 3. Transition $\gamma_{2}[(9 / 2,5)-(5 / 2,3)]$ as seen in triple resonance.

- J. C. Zorn, T. C. English, J. T. Dickinson, and D. A. Stephenson, J. Chem. Phys. 45, 3731 (1966). 
TABLE I. Triple resonances observed in $\mathrm{CsF} . \mathbf{a}, \mathrm{b}$

\begin{tabular}{|c|c|c|c|c|c|c|}
\hline \multirow[b]{3}{*}{ Line } & \multicolumn{4}{|c|}{ Transitions occurring in Field 2} & \multirow{2}{*}{\multicolumn{2}{|c|}{$\begin{array}{l}\text { Transitions occurring } \\
\text { in Fields } 1 \text { and } 3\end{array}$}} \\
\hline & & \multirow{2}{*}{$\begin{array}{l}\text { Amplitude } \\
\text { (units of } \\
10^{-15} \mathrm{~A} \text { ) }\end{array}$} & \multirow{2}{*}{$\begin{array}{l}\text { Observed } \\
\text { frequency }\end{array}$} & \multirow{2}{*}{$\begin{array}{l}\text { Frequency predicted } \\
\text { from single- } \\
\text { resonance data }\end{array}$} & & \\
\hline & $\left(F_{1}, F\right)-\left(F_{1}^{\prime}, F^{\prime}\right)$ & & & & Field 1 & Field 3 \\
\hline$\gamma_{1}$ & $(9 / 2,5)-(9 / 2,4)$ & 5 & $21.35(25)^{*}$ & $21.1(2)$ & 228.1 & 245.9 \\
\hline$\gamma_{2}$ & $(9 / 2,5)-(5 / 2,3)$ & 8 & $89.4(2)$ & $89.67(25)$ & 228.1 & 317.6 \\
\hline$\gamma_{3}$ & $(9 / 2,4)-(5 / 2,3)$ & 4 & $68.2(3)$ & $68.6(2)$ & 245.9 & 317.6 \\
\hline$\gamma_{4}$ & $(9 / 2,4)-(5 / 2,2)$ & 6 & $59.2(2)$ & $59.1(2)$ & 245.9 & 305.1 \\
\hline$\gamma_{5}$ & $(5 / 2,2)-(5 / 2,3)$ & 4 & $8.8(3)^{*}$ & $9.5(2)$ & 305.1 & 317.6 \\
\hline$\gamma_{0}$ & $(7 / 2,4)-(7 / 2,3)$ & 2 & $2.8(2)^{*}$ & $3.25(30)$ & 245.9 & 317.6 \\
\hline
\end{tabular}

${ }^{a}$ All frequencies in $\mathbf{k H z}$.

b Numbers in parentheses are uncertainties in units of the last digit.

- All transitions observed with a dc electric field of $1.5 \mathrm{~V} / \mathrm{cm}$ except

of state-selected molecules in such numbers that the most intense single resonance transitions are seen as a change of about $5 \times 10^{-14} \mathrm{~A}$ on a background of $2 \times 10^{-12}$ $\mathrm{A}$; the triple-resonance signals are from $10 \%-50 \%$ of the single-resonance amplitude. Spectrometer noise makes small signals $\left(<2 \times 10^{-15}\right.$ A) difficult to measure reliably.

With the present apparatus, one convenient method to detect resonances is to measure the change in detector current, or "flop," which is correlated with the application and removal of the rf excitation to Field 3; the application of $\omega_{3}$, a known resonance frequency, will always give a transition signal, so the $\omega_{2}$ resonance can then be detected as a decrease in the flop correlated with $\omega_{3}$. Figure 3 shows the flop from $\omega_{3}$ vs $\omega_{2}$, plotted as $\omega_{2}$ goes through the resonance frequency of the $(9 / 2,5)-$ $(5 / 2,3)$ transition.

The single resonance experiments done with the present spectrometer use a dc electric field strength of $1.5 \mathrm{~V} / \mathrm{cm}$, and an rf signal which is on the order of $\frac{1}{2} \mathrm{~V} / \mathrm{cm} \mathrm{rms}$ over the cross section of the beam. In all these measurements the total magnetic field over the entire resonance region is reduced below $0.05 \mathrm{G}$ with a set of coils. The single resonance experiments have shown that the Zeeman effect, and the dc and rf Stark effect at these field strengths can almost be neglected in the analysis of results. In order to observe some of the triple resonance signals with the spectrometer, however, it is necessary to increase the size of transitions by using a dc field strength of $3.0 \mathrm{~V} / \mathrm{cm}$ and to excite fields 1 and 3 with about twice the rf amplitude normally used with the single resonance method. Figure 3 shows that the center of the $\omega_{2}$ resonance does not seem to be shifted when the rf amplitude of $\omega_{1}$ and $\omega_{3}$ are increased if $\omega_{2}$ remains at the normal level.

It is worth noting that the three frequencies are seen sequentially by the molecules, so there should be no reason to confuse the triple resonance signals with those which might occur through the simultaneous interaction of two or more rf quanta. Several tests have been made to insure the validity of this view: interchanging the frequencies applied to fields 1 and 3 still transitions with frequencies indicated by an asterisk which were observed at $3.0 \mathrm{~V} / \mathrm{cm}$.

d Reference 1.

permitted the observation of the characteristic resonance expected in rf field 2 . On the other hand, an interchange of the frequencies applied to fields 1 and 2 , or of those applied to 3 and 2 , yielded no resonance of the $f-g$ type. In addition, a careful search for the $\gamma_{1}$ and $\gamma_{5}$ transitions proved fruitless unless both $\omega_{1}$ and $\omega_{3}$ were set to the appropriate frequencies in accord with our model for the transition process.

It should be noted that frequencies $\omega_{1}$ and $\omega_{3}$ are different for all data presented here. If one had $\omega_{1}=\omega_{3}$, then interference would be expected. This has been seen with $\mathrm{RbF}$ in the present apparatus: A typical Ramsey resonance pattern was observed when fields 1 and 3 were driven by the same oscillator while no rf excitation was applied to field 2 .

\section{RESULTS}

The results of the measurements are shown in Table I. The internal consistency of the triple resonance data is very good: for example, we expect $\gamma_{1}+\gamma_{4}+\gamma_{5}=\gamma_{2}$; we find $\gamma_{1}+\gamma_{4}+\gamma_{5}=(89.35 \pm 0.6) \mathrm{kHz}$, and $\gamma_{2}=$ $(89.4 \pm 0.2) \mathrm{kHz}$. Comparison of the single and triple resonance data shows some differences which are more than can be accounted for by the uncertainty in determining the centers of the spectral lines. However, from the single-resonance experiments, we know that the apparent center of a very-weak-field spectral line (which actually has many unresolved Stark components) may shift several hundred Hertz as the dc field is changed from $1.5-$ to $3.0 \mathrm{~V} / \mathrm{cm}$. From Table I we see that the significant discrepancies occur for lines taken at the larger $\mathrm{dc}$ field of $3.0 \mathrm{~V} / \mathrm{cm}$. Thus we conclude that the discrepancies are due to the Stark effect.

A quantitative understanding of the electric field's effect on the frequency of the $\omega_{2}$ resonances (and of the amplitudes of these resonances) requires a better knowledge of the unresolved components of the lines than we have available. The calculation of the transition probability for the very-weak-field case is difficult because the many-level system is subject to a fairly large perturbation by an rf field whose amplitude and 
polarization are imperfectly known. Another difficulty in understanding the observed lines arises because we do not know the relative populations of the Stark sublevels in the beam: the expected distribution over the sublevels is often severely and uncontrollably altered by diabatic transitions in the regions between the state selectors and the $C$ field.

\section{DISCUSSION}

The principal result of this experiment, in which the triple resonance method is demonstrated, is the measurement of spectral lines in $\mathrm{CsF}$ which are not ordinarily seen with MBER spectroscopy. For future work the triple resonance method may offer other advantages.

(1) The analysis of spectra from molecules in higher rotational states, or from molecules in which more than one nucleus shows a quadrupole interaction, is facilitated if many spectral lines can be observed and positively identified. For example, it appears that the use of triple resonance will approximately double the number of transitions which can be measured in the very-weakfield spectrum of the $J=2$ state of ${ }^{85} \mathrm{Rb}^{19} \mathrm{~F}$.

(2) The spectroscopy of $J \gg 1$ states with the MBER method may be hampered because the change in effective electric dipole moment which accompanies a single $\left|\Delta M_{J}\right|=1$ transition may not be large enough to give adequate rejection in the state selector. By a suitable choice of frequencies in the three rf fields, the net change in $\left|M_{J}\right|$ can be increased and this will give a corresponding increase in the change of effective moment. In this respect the method has an analogy with the magnetic resonance beam experiment of Cederberg and Ramsey.

An interesting feature is that spectral lines with central frequencies of $2.8 \mathrm{kHz}$ are observed with a width of $1 \mathrm{kHz}$ in this experiment. We believe these to be the lowest frequency transitions that have been seen as spectral lines by the interaction of isolated molecules with an electromagnetic field.

\footnotetext{
${ }^{7}$ J. Cederberg and N. F. Ramsey, Phys. Rev. 135, A39 (1964).
}

THE JOURNAL OF CHEMICAL PHYSICS

VOL UME 47 , N UM B ER 10

15 NOVEM B E R 1967

\title{
$N$ Representability of Fermion Density Matrices*
}

\author{
KARL F. FREED $\dagger$ \\ Department of Chemistry, Harvard University, Cambridge, Massachusetts
}

(Received 2 December 1966)

\begin{abstract}
The proof of Smith, which gives the solution to the $N$-representability problem of the first-order density matrix for the case of $N$ even and a nondegenerate energy eigenstate, is also shown to be valid for certain linear combinations of degenerate states. A connection is shown between these proper linear combinations and the eigenfuctions of a perturbed Hamiltonian for which all of the degeneracies have been lifted. For the case of $S=0$, all the eigenfunctions of the $p$ th-order density matrix (natural $p$ functions) can be taken to be eigenfunctions of total spin

$$
\left(\sum_{i=1}^{p} S_{i}\right)^{2}
$$

and if orbital angular momentum is a constant of the motion, these natural $p$ functions are eigenfunctions of the total orbital angular momentum for $L=0$. Simple wavefunctions are presented for which the natural 2 functions are not all eigenfunctions of the total pair spin. It is then shown that for $M_{S}=0$ (any integral spin) or for $L=1, M_{L}=0$, the natural 2 functions are eigenfunctions of $\left(S_{1}+S_{2}\right)^{2}$ and $\left(L_{1}+L_{2}\right)^{2}$, respectively.
\end{abstract}

\section{INTRODUCTION}

In the last few years there has been considerable interest in the properties of reduced density matrices. ${ }^{1-13}$

\footnotetext{
* This research is supported by the National Institutes of Health.

t National Science Foundation Predoctoral Fellow. Present address: Department of Theoretical Physics, The University of Manchester, England.

${ }_{1}$ P.-O. Löwdin, Phys. Rev. 97, 1474, 1490, 1509 (1955).

2 P.-O. Löwdin, Advan. Chem. Phys. 2, 207 (1959).

${ }^{3}$ R. McWeeny, Rev. Mod. Phys. 32, 335 (1960); R. McWeeny and Y. Mizuno, Proc. Roy. Soc. (London) A259, 554 (1961).

D. ter Haar, Rept. Progr. Phys. 24, 304 (1961).

${ }^{5}$ B. C. Carlson and J. M. Keller, Phys. Rev. 121, 659 (1961).

${ }^{\circ}$ A. J. Coleman, Rev. Mod. Phys. 35, 668 (1963).

7 T. Ando, Rev. Mod. Phys. 35, 690 (1963).

${ }^{8}$ C. Garrod and J. K. Percus, J. Math. Phys. 5, 1756 (1964).
}

There still remains the interesting problem of deciding whether or not the properties of a many-particle (here we are concerned with fermions only) system may be accurately calculated without the necessity of calculating or approximating the wavefunction for the $N$-particle system. For systems with only one- and two-body forces, the two-particle density matrix (the

\footnotetext{
${ }^{9}$ G. C. Hall, L. L. Jones, D. Rees, Proc. Roy. Soc. (London) A283, 194 (1965).

${ }^{10}$ F. Sasaki, Phys. Rev. 138, B1338 (1965).

it W. Kutzelnigg and V. H. Smith, Jr., J. Chem. Phys. 42, 279 (1965).

${ }_{12}$ D. W. Smith, J. Chem. Phys. 43, S258 (1965).

${ }^{13}$ T. B. Grimley and F. D. Peat, Proc. Phys. Soc. (London) 86, 249 (1965).
} 